\title{
Nonsplit domsaturation number of a graph
}

\author{
Y. Therese Sunitha Mary * and R. Kala ** \\ *Department of Mathematics, St. Xavier's College \\ Palayamkottai 627 002, Tamil Nadu, India \\ **Department of Mathematics, Manonmaniam Sundaranar University
} Tirunelveli 627 012, Tamil Nadu, India

The nonsplit domsaturation number of a graph $G, d s_{n s}(G)$ is the least positive integer $k$ such that every vertex of $G$ lies in a nonsplit dominating set of cardinality $k$.In this paper, we obtain certain bounds for $d s_{n s}(G)$ and characterize the graphs which attain these bounds.

\section{Introduction}

By a graph $G=(V, E)$ we mean a finite, undirected graph without loops or multiple edges.The order and size of $G$ are denoted by $p$ and $q$ respectively. For graph theoretical terms we refer to Harary [6] and for terms related to domination we refer Haynes et al.[7]

A subset $D$ of $V$ is said to be a dominating set in $G$ if every vertex in $V-D$ is adjacent to at least one vertex in $D$.

Kulli and Janakiram introduced the concept of nonsplit domination in graphs [9]. A dominating set $D$ of a graph $G$ is a nonsplit dominating set if $\langle V-D\rangle$ is connected. The nonsplit domination number $\gamma_{n s}(G)$ of $G$ is the minimum cardinality of a nonsplit dominating set. A nonsplit dominating set with cardinality $\gamma_{n s}(G)$ is called a $\gamma_{n s}$-set.

Acharya[1] introduced the concept of domsaturation number of a graph. The least positive integer $k$ such that every vertex of $G$ lies in a dominating set of cardinality $k$ is called the domsaturation number of $G$ and is denoted by $d s(G)$. A detailed study of this parameter was already done by Arumugam and Kala[2]. In this paper, we define nonsplit domsaturation number of a graph. We determine the value of this parameter for several classes of graphs, obtain bounds for this parameter and also characterize the graphs which attain these bounds.

\section{Main Results}

Example 2.1 (i) If $G \cong K_{p}$ then $d s_{n s}(G)=1$.

(ii) If $G \cong K_{m, n}(2 \leq m \leq n)$ then $d s_{n s}(G)=2$.

Proposition 2.2 For any connected graph $G, \gamma_{n s}(G) \leq p-1$. Further equality holds if and only if $G$ is a star.

Proof. Every set $S \subseteq V(G)$ with $|S|=p-1$ is a nonsplit dominating set of $G$ and so $\gamma_{n s}(G) \leq p-1$.

If $G$ is a star, clearly $\gamma_{n s}(G)=p-1$. Suppose $\gamma_{n s}(G)=p-1$. If $G$ is not a star, then $G$ has an edge $e=u v$ such that both $u$ and $v$ are non - pendent vertices. Now $V(G)-\{u, v\}$ is a nonsplit dominating set of $G$ and so $\gamma_{n s}(G) \leq p-2$ which is a contradiction. Hence $G$ is a star.

Corollary 2.3 For any graph $G, \gamma_{n s}(G)=p-1$ if and only if $G$ is a galaxy.

Proposition 2.4 For any graph $G, \gamma_{n s}(G) \leq d s_{n s}(G) \leq \min \left\{\gamma_{n s}(G)+\Delta(G), p-1\right\}$ and these bounds are sharp. 
Proof. Lower bound is obvious. Suppose $d s_{n s}(G)=\gamma_{n s}(G)+\Delta(G)+k$, where $k \geq 1$. Then there exists a vertex $v \in V(G)$ such that the minimum cardinality of a nonsplit dominating set $A$ containing $v$ is $\gamma_{n s}(G)+\Delta(G)+k$. If $S$ is any $\gamma_{n s}$ - set, then $v \notin S$. Also $S \cap N(v) \neq \varnothing$. As $|A|=\gamma_{n s}(G)+\Delta(G)+k$, by choice of $v,\langle V-(S \cup\{v\})\rangle$ has $\Delta(G)+k-1$ isolated vertices so that $|N(v)| \geq \Delta(G)+k$, which is a contradiction. Hence $d s_{n s}(G) \leq \gamma_{n s}(G)+\Delta(G)$. Always $d s_{n s}(G) \leq p-1$ and so $d s_{n s}(G) \leq \min \left\{\gamma_{n s}(G)+\Delta(G), p-1\right\}$.

If $G \cong C_{p}, d s_{n s}(G)=\gamma_{n s}(G)=p-2$ and so the lower bound is sharp. If $G \cong B(2,2)$, then $d s_{n s}(G)=5$ and $\min \left\{\gamma_{n s}(G)+\Delta(G), p-1\right\}=\min \{7,5\}=5$. Thus the upper bound is also sharp.

Theorem 2.5 Let $G$ be a connected graph. Then $d s_{n s}(G)=p-1$ if and only if $G \cong G_{i}(1 \leq i \leq 2)$ where $G_{i}(1 \leq i \leq 2)$ are given in Fig. 1 .

$(2,-1)(3,2)[$ dotscale $=1](-2.3,0)(-2,-2)(0,-1.5)(-4.3,-1.5)(6,0)(4,-2)(3,-4)(5,-4.3)(6,-2)(5.5,-4.1)(7.5$, $-4.3)(8,-1.7)(9.2,-3.4)(9.5,-1)(4.6,1)(7,1)(-4.3,-1.5)(-2.3,0)(-2,-2)(-2.3,0)(0,-1.5)(6,0)(4,-2)(3,-4)(4$, $-2)(5,-4.3)(6,0)(6,-2)(5.5,-4.1)(6,-2)(7.5,-4.3)(6,0)(8,-1.7)(9.2,-3.4)(8,-1.7)(9.5,-1)(4.6,1)(6,0)(7,1)$ $[$ dotscale $=.65](-1.5,-1.9)(-1,-1.8)(-.5,-1.64)[$ dotscale $=.65](5.1,1)(5.8,1)(6.5,1)[$ dotscale $=.65](9.275$, $-2.9)(9.35,-2.2)(9.425,-1.5)$ [dotscale $=.65](3.5,-4.075)(4,-4.15)(4.5,-4.225)$ [dotscale $=.65](6,-4.15)(6.5$, $-4.2)(7,-4.25)[$ dotscale $=.65](6.5,-1.9)(7,-1.8)(7.5,-1.7)$

Proof. If $d s_{n s}(G)=p-1$ then there exists at least one vertex $v \in V(G)$ such that the only minimal nonsplit dominating set containing $v$ is of cardinality $p-1$.

Case(i) : $v$ is a pendent vertex.

In this case, we have $\gamma_{n s}(G)=p-1$ by choice of $v$. Hence by Proposition $2.1 G \cong G_{1}$.

Case (ii) $: v$ is a non-pendent vertex.

Let $N(v)=\left\{v_{1}, v_{2}, \ldots, v_{k}\right\}(k \geq 2)$. If there exists an edge $\left(v_{i}, v_{j}\right) \in<N(v)>,(1 \leq i, j \leq k)$ then $V(G)-\left\{v_{i}, v_{j}\right\}$ is a nonsplit dominating set containing $v$ and so $\langle N(v)\rangle$ is independent.

We now claim that every vertex in $V(G)-N[v]$ is a pendent vertex. Suppose there exists $u \in V(G)-N[v]$ such that $d(u) \geq 2$. Since $G$ is connected, there exists a $u-v$ path $P$ with length at least 2. Let $w \in N(u) \cap P$. Then $V(G)-\{u, w\}$ is a nonsplit dominating set containing $v$ and hence $G \cong G_{2}$.

Converse is obvious.

The following is immediate.

Corollary 2.6 Let $G$ be any graph. Then $d s_{n s}(G)=p-1$ if and only if every component of $G$ is isomorphic to any one of the graphs in Fig. 1.

Theorem 2.7 For any tree $T, d s_{n s}(\bar{T})=\gamma_{n s}(\bar{T})=2$ if and only if $T$ is not isomorphic to $B(r, s)$ where at least one of $r$ or $s$ equals 1.

Proof. Suppose $T \cong B(r, s)$ where $r=s=1$. Then $T \cong P_{4}$ and $\gamma_{n s}\left(\overline{P_{4}}\right)=2$. But $d s_{n s}\left(\overline{P_{4}}\right)=3$. Hence $T \circledast B(r, s)$ where $r=s=1$. If $T \cong B(r, s)$ with exactly one of $\{r, s\}$ having value 1 , then there is no $\gamma_{n s}$-set of $\bar{T}$ of cardinality 2 containing $u$. These contradictions exhibit that $T$ is not isomorphic to $B(r, s)$ where at least one of $r$ and $s$ equals 1 .

Conversely assume that $T$ is a tree not isomorphic to $B(r, s)$ where at least one of $r$ and $s$ equals 1 . If $T \cong K_{1, p-1}$ then $\gamma_{n s}(\bar{T})=2=d s_{n s}(\bar{T})$. If $T \AA K_{1, p-1}$, then there exists at least 2 pendent vertices $u$ and 
$v$ with distinct supports $u_{1}$ and $v_{1}$ respectively such that $\operatorname{deg}\left(u_{1}\right) \leq p-3$ and $\operatorname{deg}\left(v_{1}\right) \leq p-3$.

Case(i) : $\operatorname{deg}\left(u_{1}\right)=p-3$ and $\operatorname{deg}\left(v_{1}\right)=p-3$.

If $u_{1}$ and $v_{1}$ are adjacent then $T \cong T_{1}$ where $T_{1}$ is given in Fig. 2 .

$(3,-1)(3,1)$ dotscale $=1.5](0,0)(2,0)(-1.5,-1)(-1.5,1)(3.5,1)(3.5,-1)(-1.5,1)(0,0)(2,0)(3.5,1)(-1.5,-1)(0,0)$ $(2,0)(3.5,-1)$

$\left\{v, v_{1}\right\},\left\{v_{2}, v_{1}\right\},\left\{u, u_{1}\right\},\left\{u_{2}, u_{1}\right\}$ are all minimum nonsplit dominating sets of $\bar{T}$ and so $\gamma_{n s}(\bar{T})=d s_{n s}(\bar{T})=2$. If $u_{1}$ and $v_{1}$ are non-adjacent then $T \cong P_{5}$ and so $\gamma_{n s}(\bar{T})=d s_{n s}(\bar{T})=2$.

Case(ii) : $\operatorname{deg}\left(u_{1}\right)=p-3$ and $\operatorname{deg}\left(v_{1}\right) \neq p-3$.

If $u_{1}$ and $v_{1}$ are adjacent, then $T \cong T_{2}$ where $T_{2}$ is given in Fig. 3 .

$(-1,-1)(3,0)$ [dotscale $=1.5](-2,0)(2,0)(2,-1.5)(-1,-1.5)(-3,-1.5)(3.5,-.7)(.2,-1.5)(2,-1.5)(2,0)(-2,0)(-1,-1.5)$

$(-2,0)(-3,-1.5)(2,0)(3.5,-.7)(-2,0)(.2,-1.5)[$ dotscale $=.65](-.7,-1.5)(-.4,-1.5)(-.1,-1.5)$

Since $d\left(v_{1}\right) \neq p-3, d\left(v_{1}\right) \geq 4$. For every $u^{\prime} \in N\left(u_{1}\right),\left\{u_{1}, u^{\prime}\right\}$ is a $\gamma_{n s}-$ set of $\bar{T}$ and for every $v^{\prime} \in N\left(v_{1}\right),\left\{v_{1}, v^{\prime}\right\}$ is a $\gamma_{n s}$-set of $\bar{T}$ and so $\gamma_{n s}(\bar{T})=d s_{n s}(\bar{T})=2$. If $u_{1}$ and $v_{1}$ are non-adjacent then $T \cong T_{3}$ where $T_{3}$ is given in Fig. 4 .

$(-1.5,-1)(3, .5)[$ dotscale $=1.5](-2,0)(0,0)(2,0)(2,-1.5)(-1,-1.5)(-3,-1.5)(2,-1.5)(2,0)(0,0)(-2,0)(-1,-1.5)(-2$, $0)(-3,-1.5)$ [dotscale $=.65](-1.5,-1.5)(-2,-1.5)(-2.5,-1.5)$

As above $\operatorname{deg}\left(u_{1}\right) \geq 3$. For every $u^{\prime} \in N\left(u_{1}\right),\left\{u^{\prime}, u_{1}\right\}$ is a $\gamma_{n s}$ - set of $\bar{T}$. Also $\left\{u_{1}, v\right\}$ and $\left\{v_{1}, u\right\}$ are $\gamma_{n s}$ set of $\bar{T}$ and so $\gamma_{n s}(\bar{T})=d s_{n s}(\bar{T})=2$.

Case(iii) : $\operatorname{deg}\left(u_{1}\right) \neq p-3$ and $\operatorname{deg}\left(v_{1}\right)=p-3$.

This is analogous to case(ii).

Case(iv): $\operatorname{deg}\left(u_{1}\right) \neq p-3$ and $\operatorname{deg}\left(v_{1}\right) \neq p-3$.

If $u_{1}$ and $v_{1}$ are adjacent then $\operatorname{deg}\left(u_{1}\right) \geq 4$ and $\operatorname{deg}\left(v_{1}\right) \geq 4$ and for every $u^{\prime} \in N\left(u_{1}\right),\left\{u_{1}, u^{\prime}\right\}$ is a $\gamma_{n s}$-set of $\bar{T}$ and for every $v^{\prime} \in N\left(v_{1}\right),\left\{v_{1}, v^{\prime}\right\}$ is a $\gamma_{n s}$-set of $\bar{T}$ so that $d s_{n s}(\bar{T})=\gamma_{n s}(\bar{T})=2$.

Suppose $u_{1}$ and $v_{1}$ are non-adjacent. Then $\operatorname{deg}\left(u_{1}\right) \geq 3$ and $\operatorname{deg}\left(v_{1}\right) \geq 3$. For every $x \in V(T)$ with $d\left(u_{1}, x\right) \neq 2,\left\{x, u_{1}\right\}$ is a $\gamma_{n s}$-set of $\bar{T}$ containing $x$ and if $d\left(u_{1}, x\right)=2,\{x, u\}$ is a $\gamma_{n s}$-set of $\bar{T}$ containing $x$. The $\gamma_{n s}$-sets containing neighbours of $u_{1}$ and $v_{1}$ are as above. Thus $d s_{n s}(\bar{T})=\gamma_{n s}(\bar{T})=2$.

Theorem 2.8 There exists a graph $G$ for which $d s_{n s}(G)-d s(G)$ can be made arbitrarily large.

Proof. Let $P_{p-k}=\left\{u_{1}, u_{2}, \ldots, u_{p-k}\right\}$ be a path on $p-k$ vertices where $1 \leq k \leq p-1$ and let $S=\left\{v, v_{1}, v_{2}, \ldots, v_{k-1}\right\}$. Join the vertex $v$ to each of the vertices in $P_{p-1}$ and to each vertex in $S-\{v\}$. The resulting graph $G$ is of order $p$ and $\gamma(G)=1$. Also $\left\{v, u_{i}\right\}(1 \leq i \leq p-k)$ and $\left\{v, v_{j}\right\}(1 \leq j \leq k-1)$ are minimal dominating sets containing $u_{i}, u_{j}$ respectively so that $d s(G)=2$.

$S$ is a minimum nonsplit dominating set of $G$ and so $\gamma_{n s}(G)=k$. If $k=p-1$ or $p-2$, $d s_{n s}(G)=k$. Suppose $k \leq p-3 . S \cup\left\{u_{1}\right\} \quad, \quad S \cup\left\{u_{p-k}\right\} \quad, \quad(S-\{v\}) \cup\left\{u_{2}, u_{5}, \ldots\right\}$, $(S-\{v\}) \cup\left\{u_{1}, u_{3}, u_{6}, \ldots\right\}$ and $(S-\{v\}) \cup\left\{u_{1}, u_{4}, u_{7}, \ldots\right\}$ are all nonsplit dominating sets of $G$ and so 
$d s_{n s}(G)=k+\left\lfloor\frac{p-k}{3}\right\rfloor$ or $k+\left\lceil\frac{p-k}{3}\right\rceil$ according as $p-k \equiv 0,1(\bmod 3)$ or $p-k \equiv 2(\bmod 3)$. Thus $d s_{n s}(G)-d s(G)=k+\left\lfloor\frac{p-k}{3}\right\rfloor-2$ or $k+\left\lceil\frac{p-k}{3}\right\rceil-2$ where $k$ can be chosen arbitrarily large.

Theorem 2.9 For any connected graph $G, d s_{n s}(G)+\operatorname{diam}(G) \leq 2 p-2$ and equality holds if and only if $G \cong P_{p}(p \leq 5)$.

Proof. Since $G \quad$ is connected, $\operatorname{diam}(G) \leq p-1$. Always $d s_{n s}(G) \leq p-1 \quad$ and $\quad$ so $d s_{n s}(G)+\operatorname{diam}(G) \leq 2 p-2$. Suppose $d s_{n s}(G)+\operatorname{diam}(G)=2 p-2$. Then $d s_{n s}(G)=p-1$ and $\operatorname{diam}(G)=p-1$. Since $d s_{n s}(G)=p-1$, by Theorem ?? we observe that $\operatorname{diam}(G) \leq 4$ and so $p \leq 5$. For any graph on $p$ vertices other than $P_{p}$ we have $d s_{n s}(G)+\operatorname{diam}(G) \neq 2 p-2$ and so $G \cong P_{p}(p \leq 5)$. Converse is obvious.

Theorem 2.10 For any connected graph $G$ with at least two pendent vertices, $d s(G)+d s(\bar{G}) \leq d s_{n s}(G)+d s_{n s}(\bar{G}) \leq p+2$. Also the bounds are sharp.

Proof. For $\quad$ any $\quad$ graph $\quad G \quad, \quad d s(G) \leq d s_{n s}(G) \quad, \quad d s(\bar{G}) \leq d s_{n s}(\bar{G}) \quad$ and $\quad$ so $d s(G)+d s(\bar{G}) \leq d s_{n s}(G)+d s_{n s}(\bar{G})$. Always $d s_{n s}(G) \leq p-1$. To establish the upper bound it is enough to prove that $d s_{n s}(\bar{G}) \leq 3$. Let $P=\left\{u_{1}, u_{2}, \ldots, u_{m}\right\}$ be the set of pendent vertices of $G$ and $S=\left\{v_{i}(1 \leq i \leq m)\right\}$ be the set of corresponding supports (not necessarily distinct). If $m \geq 3$ and there exists an index $i$ such that $\left\{V(G)-\left\{u_{i}, v_{i}\right\}\right\}$ has two distinct supports then $A=\left\{u_{i}, v_{i}\right\}$ is a nonsplit dominating set of $\bar{G}$. If $w$ is the unique support in $\left\langle V(G)-\left\{u_{i}, v_{i}\right\}\right\rangle$ then $\left\{u_{i}, v_{i}, w\right\}$ is a nonsplit dominating set of $\bar{G}$. Otherwise $v_{i}$ is the only support of $G$ and $A=\left\{u_{i}, v_{i}\right\}$ is a nonsplit dominating set of $\bar{G}$. For every other vertex $x, A \cup\{x\}$ is nonsplit dominating set of $\bar{G}$. Hence $d s_{n s}(\bar{G}) \leq 3$.

Suppose $m=2$. Let the two pendent vertices be $u$ and $v$ with supports $u_{1}$ and $v_{1}$ respectively.

Case(i) : $u_{1}=v_{1}$

Let $D=V(G)-\left\{u, v, u_{1}\right\}$. If $D=\phi$ then $\left\{u, v_{1}\right\}$ and $\left\{v, v_{1}\right\}$ are nonsplit dominating sets of $\bar{G}$. If $D \neq \phi$ then $\left\{u, v_{1}\right\},\left\{v, v_{1}\right\}$ and $\left\{u, v_{1}, x\right\}$ [where $\left.x \in V(G)-\left\{u, v, u_{1}\right\}\right]$ are nonsplit dominating sets of $\bar{G}$.

Case(ii) : $u_{1} \neq v_{1}$.

If $\left(u_{1}, v_{1}\right) \notin E(G)$ then $\left\{v, u_{1}\right\},\left\{u, v_{1}\right\}$ and $\left\{u, v_{1}, x\right\}$ [where $\left.x \in V(G)-\left\{u, v, u_{1}, v_{1}\right\}\right]$ are nonsplit dominating sets of $\bar{G}$. Suppose $\left(u_{1}, v_{1}\right) \in E(G)$ and let $B=V(G)-\left\{u, v, u_{1}, v_{1}\right\}$. If $B=\phi$ then $\left\{v, v_{1}, u_{1}\right\}$ and $\left\{v_{1}, u_{1}, u\right\}$ are nonsplit dominating sets of $\bar{G}$. Suppose $B \neq \varnothing$. If $|B| \geq 2$ then $\left\{v, v_{1}\right\},\left\{u, u_{1}\right\},\left\{x, u_{1}\right\}(x \in B)$ are nonsplit dominating sets of $\bar{G}$. If $|B|=1$ and $B=\{w\}$ then $\left\{u_{1}, v_{1}, w\right\},\left\{u, u_{1}, v_{1}\right\},\left\{u_{1}, v_{1}, v\right\}$ are nonsplit dominating sets of $\bar{G}$. Hence $d s_{n s}(\bar{G}) \leq 3$. Thus $d s_{n s}(G)+d s_{n s}(\bar{G}) \leq p+2$.

Lower bound is attained for $K_{2}$ and upper bound for $P_{4}$. Hence the bounds are sharp.

Definition 2.11 Let $G=(V, E)$ be a graph. The maximum order of a partition of $V$ into nonsplit 
dominating sets of $G$ is called the nonsplit domatic number of $G$ and is denoted by $d_{n s}(G)$.

Definition 2.12 A graph $G$ with $d_{n s}(G)=\delta(G)+1$ is said to be nonsplit domatically full.

Theorem 2.13 If $G$ is a $k$-regular graph which is nonsplit domatically full then $\gamma_{n s}(G)=d s_{n s}(G)$.

Proof. Since $G$ is nonsplit domatically full, $d_{n s}(G)=k+1$. Let $\left\{D_{1}, D_{2}, \ldots, D_{k+1}\right\}$ be a nonsplit domatic partition of $G$. Any set $D_{i}$ either contains a vertex $u$ or exactly one of its neighbours. Hence, each $D_{i}$ is independent. Also, for all $1 \leq j \leq k+1, i \neq j$, every vertex in $D_{i}$ is adjacent to exactly one vertex in $D_{j}$. Hence all sets $D_{i}$ are of equal cardinality and $\left|D_{i}\right|=\gamma_{n s}(G)$. Hence $\gamma_{n s}(G)=d s_{n s}(G)$.

Remark 2.14 The converse of theorem 2.13 is not true. The 3-regular graph $G$ given in Fig.5 is not nonsplit domatically full.

$(-5,-1)(3,2)$ [dotscale $=1.5](-3.5,0)(4,0)(-1.5,1)(1.5,1)(-1.5,-1)(1.5,-1) \quad(-3.5,0)(4,0)(-3.5,0)(-1.5,1)(1.5$, 1)(4, 0) $(-3.5,0)(-1.5,-1)(1.5,-1)(4,0)(-1.5,1)(-1.5,-1)(1.5,1)(1.5,-1)$

We observe that $d s_{n s}(G)=\gamma_{n s}(G)=2$.

\section{References}

[1]. B. D. Acharya, The strong domination number of a graph and related concepts, J.Math. Phys.Sci. 14(1980), No. 5, 471-475.

[2]. S. Arumugam and R. Kala, Domsaturation number of a graph, Indian J. Pure appl. Math., 33(2002), No. 11, 1671-1676.

[3]. J. R. Carrington, F. Harary and T. W. Haynes, Changing and unchanging the domination number of a graph, J. Combin. Math. Combin. Comput., 9(1991), 57-63.

[4]. E. J. Cockayne, Domination of undirected graphs - A survey. In theory and Applications of Graphs. LNM 642, Springer - Verlag, (1978), 141-147.

[5]. E. J. Cockayne and S. T. Hedetniemi, Towards a theory of domination in graphs, Networks, 7, (1977), $247-261$.

[6]. F. Harary, Graph theory, Addison Wesley, Reading Mass (1969).

[7]. T. W. Haynes, S. T. Hedetniemi and P. J. Slater, Fundamentals of Domination in graphs, Marcel Dekker, Inc., (1998).

[8]. T. W. Haynes, S. T. Hedetniemi and P. J. Slater, Domination in Graphs - Advanced Topics, Marcel Dekker, (1998).

[9]. V. R. Kulli and B. Janakiram, The nonsplit domination number of a graph, Indian J. Pure Appl. Math., 31 (2000), No. 4, 545-550.

[10]. Y. Therese Sunitha Mary and R. Kala, The nonsplit domination number of a graph, Proceedings of the National Conference on Mathematical and Computational Models, December 2007, PSG College of Technology, Coimbatore, India, $218-224$. 